\title{
LARGE EDDY SIMULATION OF AN ELLIPTIC JET INJECTED INTO A SUPERSONIC CROSSFLOW
}

\author{
GUO-LEI WANG AND XI-YUN LU \\ Department of Modern Mechanics, University of Science and Technology of China, \\ Hefei, Anhui 230027, China \\ xlu@ustc.edu.cn
}

\begin{abstract}
A transverse jet issuing from an elliptic injector into a supersonic crossflow has been investigated using large eddy simulation. The complex flow structures and the relevant flow features are analyzed to exhibit the evolution of shock structures, vertical structures and jet shear layer. A horseshoe vortex is formed in the upstream of the jet and the shock structures exhibit small fluctuations due to the flow interaction. The kidney-shaped counter-rotating vortex pair dominates the flow field in the downstream of the jet. The elliptic jet spreads rapidly in the spanwise direction and then the axis-switching phenomenon occurs. Intense turbulent fluctuations are identified behind the Mach disk because of the large velocity gradients.
\end{abstract}

Keywords: Large eddy simulation; elliptic jet; compressible turbulent flow.

\section{Introduction}

The flow phenomena related to the transverse jet injected into a supersonic crossflow are of importance in both fundamentals and applications. Early investigations demonstrated that the jet-to-crossflow momentum flux ratio, defined as $J=\rho_{j} U_{j}^{2} / \rho_{\infty} U_{\infty}^{2}$, where the subscript $j$ represents the jet exit conditions and $\infty$ the crossflow conditions, is a dominant parameter in the flow evolution ${ }^{1}$. Some experiment on the flow features induced by circular and elliptic injections revealed that the elliptic injection may be an effective mixing enhancement scheme ${ }^{2}$. Detailed flow structures were also obtained using planar Rayleigh/Mie scattering ${ }^{2,3}$. Although some numerical simulations for the circular injection have been performed ${ }^{4,5}$, the relevant physical flow behaviors for the elliptic injection are still unclear and are deserved to be studied.

In the present study, a transverse elliptic jet injected into a supersonic crossflow is investigated. Large eddy simulation is implemented in solving the Favre-filtered compressible Navier-Stokes equations. The motivation of this work is to study various fundamental mechanisms dictating the complex flow phenomena.

\section{Mathematical Formulation and Numerical Methods}

The governing equations are numerically solved by a finite-volume method. The convective terms are discretized by a central/upwind hybrid scheme for shock-capturing 
and the viscous terms by a fourth-order central difference ${ }^{6-8}$. Time advancement is performed by an implicit approximate-factorization method with sub-iterations to ensure a second-order accuracy. Detailed formulations are described in our previous papers ${ }^{7,8}$.

In this study, the initial condition is set as the free-stream quantities. No-slip and adiabatic conditions are applied on the wall. Far field boundary conditions at the upper and side boundaries are treated by local one-dimensional Riemann-invariants ${ }^{8}$. The jet boundary conditions are set on the surface using a specified velocity profile and constant static pressure. The density can be obtained by the Crocco-Busemann relation. The mean velocity profile obtained from RANS with small random disturbance is used as the inflow boundary conditions ${ }^{4}$.

\section{Results and Discussion}

We consider an elliptic sonic jet issuing transversely into a supersonic crossflow at $M_{\infty}=1.6$. The aspect ratio of the elliptic injector is 0.25 and its major axis is aligned with the crossflow. The effective diameter is $D_{e}=4 \mathrm{~mm}$. To compare with the circular injection case, the following parameters are the same as those used in the experiment for the circular injection ${ }^{9}$. The free-stream stagnation pressure is $p_{0 \infty}=241 \mathrm{kPa}$ and the stagnation temperature is $T_{0 \infty}=295 \mathrm{~K}$. The air jet stagnation pressure is chosen as $p_{0 j}=476 \mathrm{kPa}$ and stagnation temperature is $T_{0 j}=295 \mathrm{~K}$. The resulting jet-to-crossflow momentum ratio is $J=1.7$ and the Reynolds number defined as $R e=\rho_{\infty} U_{\infty} D_{e} / \mu_{\infty}$, is $1.38 \times 10^{5}$. Based on our careful examinations, the computational domain is set as $-5 \leq x / D_{e} \leq 20$ in the streamwise $(x)$ direction, $-10 \leq y / D_{e} \leq 10$ in the spanwise $(y)$ direction, and $0 \leq z / D_{e} \leq 15$ in the vertical ( $z$ ) direction in the coordinate system of the injector being centered at the origin, respectively.
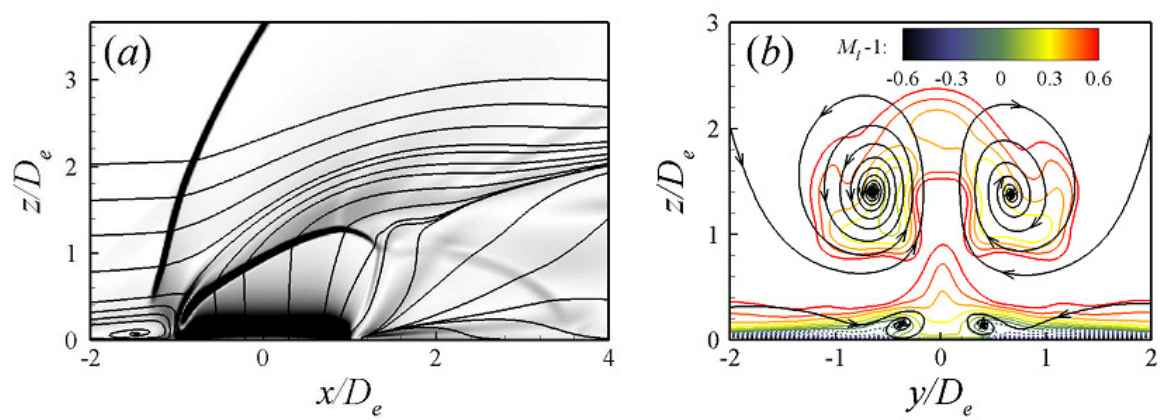

Fig. 1. Flow field patterns: (a) numerical schlieren like visualization by contours of $\|\nabla \rho\|$ and the streamlines in $y / D_{e}=0$ plane; (b) mean local Mach number $M_{l}$ distributions and the streamlines in $x / D_{e}=5$ plane, here, solid lines denoting $M_{l}>1$ and dashed lines $M_{l}<1$.

The numerical schlieren and the streamlines of the mean flow field in the $y / D_{e}=0$ plane are shown in Fig. 1(a). As a sonic jet issuing perpendicularly from an elliptic injector into the supersonic crossflow, both strong bow shock and weak separation shock are induced in the upstream of the jet. The under-expanded jet expands through the Prandtl-Meyer fan and then is compressed by a barrel shock and a Mach disk. After 
careful comparison with the flow structures of the circular injection ${ }^{10}$, we find that the strength of the bow shock indicated by its inclination to the crossflow is weaker than that in the circular injection. As observed in the experiment ${ }^{3}$, the shock structures exhibit quite small fluctuation. The streamlines originated from the injector indicate that most of the jet fluid emerges from the upper edge of the barrel shock and retains much of its transverse momentum flux ${ }^{9}$. The recirculation region depicted by the streamlines in the upstream of the jet corresponds to the horseshoe vortex which is related to the flameholding capability in combusting situation ${ }^{3}$. Upwash and downwash velocities are detected in the downstream of the jet and a node is also detected. Moreover, a weak reflected shock is observed in the downstream of the jet.

The mean streamlines and local Mach number distributions in the $x / D_{e}=5$ plane are shown in Fig. 1(b). The large-scale counter-rotating vortex pair (CVP) depicted by the streamlines dominates the flow field ${ }^{9}$. The center of rotation of the right side vortex is obtained as $\left(y / D_{e}, z / D_{e}\right)=(0.65,1.37)$, while it is detected to be $(0.54,1.30)$ for the circular injection under the same conditions. The spanwise position of the center of rotation for the elliptic injection is about $20 \%$ further than that for the circular injection. This phenomenon represents that the elliptic injection can increase the distance between the counter-rotating vortices, then more freestream fluid will be entrained into the jet core $^{2}$. The strength of these streamwise vortices (i.e. the circulation) is further analyzed. It is identified that the vortices in the elliptic injection are stronger than those in the circular injection. Relatively small-scale CVP is detected near the wall which is associated with the boundary layer separation ${ }^{5}$. Low local Mach number values are reasonably detected near the center of rotation of the vortices and near the wall. The jet plume spreads further in the spanwise direction than in the vertical direction, indicating that the axis-switching phenomenon occurs ${ }^{2}$.
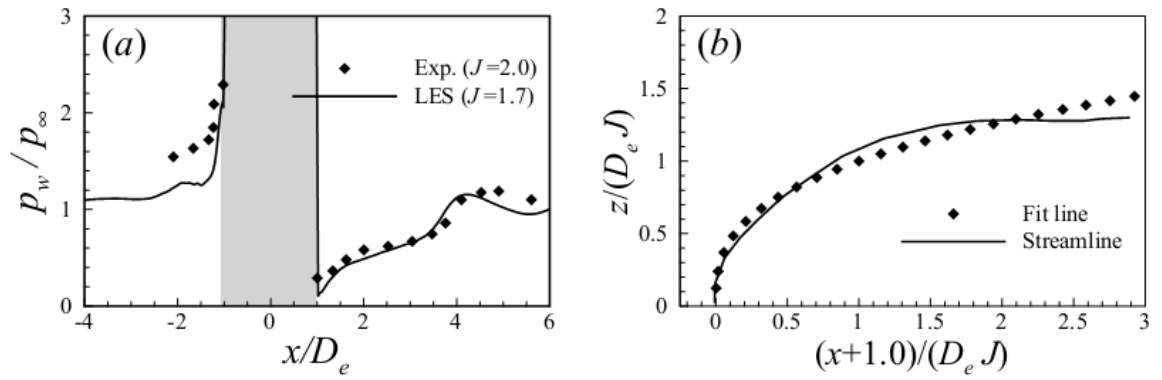

Fig. 2. Distribution of the wall pressure and jet penetration: (a) wall pressure with the experimental data in $y / D_{e}=0$ plane; (b) jet penetration depicted by the streamlines with the empirical power law curve fit based on the experimental data.

Fig. 2(a) shows the mean wall pressure distribution in the $y / D_{e}=0$ plane. The symbols denote the experimental data obtained from an elliptic jet as $J=2.0^{1}$. Due to the lower momentum flux ratio discussed here, the wall pressure in the horseshoe vortex region is somewhat lower than the experimental data. Basically, the numerical result agrees reasonably with the experimental data in the downstream of the jet. The streamline 
originated from the upstream edge of the injector is usually used to identify the jet penetration ${ }^{10}$. As shown in Fig. 2(b), the streamline agrees well with the power law curve fit defined as, $z /\left(D_{e} J\right)=1.0\left[(x+1.0) /\left(D_{e} J\right)\right]^{0.344}$, based on the experimental observation $^{2}$.
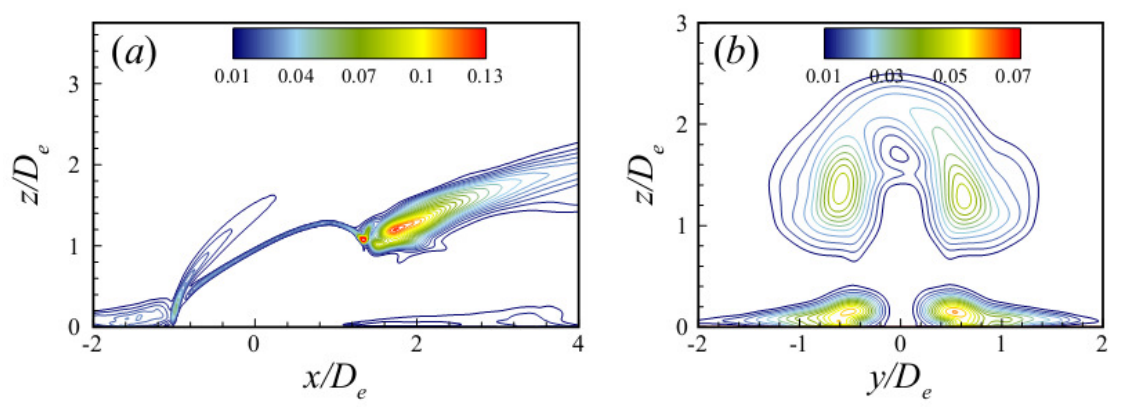

Fig. 3. Distribution of the specific turbulent kinetic energy: (a) in $y / D_{e}=0$ plane; (b) in $x / D_{e}=5$ plane.

The relevant turbulent behaviors in the flow field are further analyzed. Fig. 3(a) shows the iso-contours of the specific turbulent kinetic energy (TKE), defined as $k=\left\{u_{i}^{\prime \prime} u_{i}^{\prime \prime}\right\} / 2$. The amplification of TKE in the horseshoe vortex region due to the shock wave/boundary layer interaction is prominent in the upstream of the jet ${ }^{9}$. A jet shear layer with low TKE values is formed at the jet/freestream interface. As observed in both experiments $^{2,3}$ and numerical simulations ${ }^{4,5,10}$, large scale coherent structures are easily identified in instantaneous snapshots. As the strong upwash velocity encountering with the subsonic jet fluid after the Mach disk, high levels of TKE are detected in the downstream of the jet. It is noticed that the maximum value of TKE is around 0.13 near $x / D_{e}=2$. With the flow evolution in the downstream, turbulence rapidly becomes weak. Moreover, it is identified that the regions with high levels of TKE are consistent with the $M<1$ regions ${ }^{10}$. The iso-contours of TKE in the $x / D_{e}=5$ plane are depicted in Fig. 3(b). The characteristic kidney-shaped structures are prominent, corresponding to the counterrotating vortex pair ${ }^{9}$. High levels of TKE are detected near the wall which is related to the spanwise boundary layer separations. The maximum TKE value is detected to be 0.05 in the center of rotation of the counter-rotating vortices.

We finally analyze the Reynolds normal stress in the downstream of the jet. Fig. 4(a) exhibits the transverse distributions of the Reynolds normal stresses at $x / D_{e}=2$ in the $y / D_{e}=0$ plane. Due to the strong upwash velocity shown in Fig. 1(a), the vertical component is dominant as obtained in the circular injection ${ }^{10}$. The spanwise distribution of the Reynolds stresses near the center of rotation of the counter-rotating vortices in the $x / D_{e}=5$ plane is shown in Fig. 4(b). The Reynolds stresses have quite small values and the location of the peak value corresponds to the center of rotation of the counter-rotating vortices. 

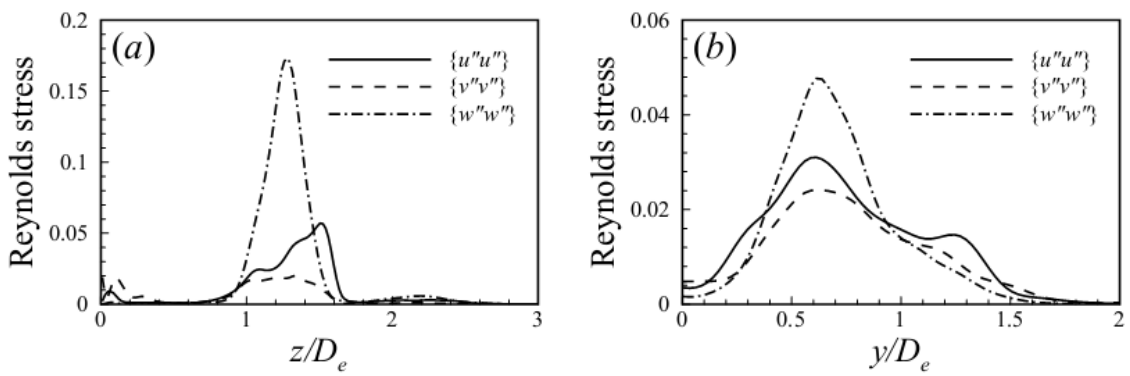

Fig. 4. Distribution of the Reynolds normal stress in the downstream of the jet: (a) transverse distribution at $x / D_{e}=2$ in $y / D_{e}=0$ plane; (b) spanwise distribution at $z / D_{e}=1.25$ in $x / D_{e}=5$ plane.

\section{Acknowledgments}

This work was supported by the National Natural Science Foundation of China (GrantNo.11072236) and the Fundamental Research Funds for the Central Universities (GrantNo.WK2090050007).

\section{References}

1. M. R. Gruber and L. P. Goss, J. Propul. Power, 15, 633 (1999).

2. M. R. Gruber, A. S. Nejad, T. H. Chen and J. C. Dutton, J. Propul. Power, 16, 449 (2000).

3. M. R. Gruber, A. S. Nejad, T. H. Chen and J. C. Dutton, AIAA J. 34, 2191 (1996).

4. D. M. Peterson and G. V. Candler, AIAA J. 26, 533 (2010).

5. S. Kawai and S. K. Lele, AIAA J. 48, 2063 (2010).

6. X.-Y. Lu, S.-W. Wang, H. G. Sung, and et al., J. Fluid Mech. 527,171 (2005).

7. L.-W. Chen, C.-Y. Xu and X.-Y. Lu, J. Fluid Mech. 643, 97 (2010).

8. C.-Y. Xu, L.-W. Chen and X.-Y. Lu, J. Fluid Mech. 665, 238 (2010).

9. J. G. Santiago and J. C. Dutton, J. Propul. Power, 13, 264 (1997).

10. G.-L. Wang and X.-Y. Lu, Theor. Appl. Mech. Lett. 1, 012005 (2011). 